\title{
Quantitative analysis of 3-Tesla magnetic resonance imaging in the differential diagnosis of breast lesions
}

\author{
ZHEN-SHEN MA ${ }^{1}$, DA-WEI WANG ${ }^{1}$, XIU-BIN SUN ${ }^{2}$, HAO SHI ${ }^{1}$, \\ TAO PANG ${ }^{1}$, GUI-QING DONG ${ }^{1}$ and CHENG-QI ZHANG ${ }^{1}$ \\ ${ }^{1}$ Department of Medical Imaging, Qianfoshan Hospital Affiliated to Shandong University, Jinan, Shandong 250014; \\ ${ }^{2}$ Department of Epidemiology and Health Statistics, School of Public Health, \\ Shandong University, Jinan, Shandong 250012, P.R. China
}

Received April 30, 2014; Accepted October 30, 2014

DOI: $10.3892 /$ etm.2014.2154

\begin{abstract}
The aim of this study was to investigate the value of quantitative 3-Tesla (3T) magnetic resonance (MR) assessment in the diagnosis of breast lesions. A total of 44 patients with breast lesions were selected. All the patients underwent MR plain scanning and T1 dynamic contrast-enhanced imaging. The vascular function parameters of the lesions, namely volume transfer constant (Ktrans), rate constant (Kep), extravascular extracellular volume fraction (Ve) and integrated area under the curve (iAUC), were acquired. These parameters were compared between benign and malignant breast lesions, and also among differential grades of invasive ductal carcinoma. The values of Ktrans, Kep and iAUC were significantly different between the benign and malignant tumors; however, the values of Ve in the benign and malignant tumors were not significantly different. The values of Ktrans, Kep and iAUC in invasive ductal carcinoma were significantly different between grade I and grade II, and between grade I and grade III; however, there was no significant difference between grade II and grade III. The Ve values in invasive ductal carcinoma did not significantly differ among grades I, II and III. Among the vascular function parameters, Ktrans exhibited the highest sensitivity and specificity in the differentiation of benign and malignant lesions. Quantitative 3-T MR assessment is valuable in the diagnosis of benign and malignant breast lesions. It can also provide reference values for the differentiation of the histological grade of breast invasive ductal carcinoma.
\end{abstract}

Correspondence to: Dr Cheng-Qi Zhang, Department of Medical Imaging, Qianfoshan Hospital Affiliated to Shandong University, 16766 Jingshi Road, Jinan, Shandong 250014, P.R. China

E-mail: zscqcn@163.com

Key words: breast lesions, dynamic contrast-enhanced magnetic resonance imaging, vascular function parameters, pathological grading, differential diagnosis

\section{Introduction}

Breast cancer is one of the most common malignant diseases endangering women's health. The prevalence of breast cancer has continued to rise in recent years, and the individuals affected have been getting progressively younger. Breast magnetic resonance imaging (MRI) has high sensitivity and specificity (1,2). In the 1970s, Folkman (3), a professor from Harvard University, proposed that tumor growth and metastasis depended on angiogenesis, and since then, numerous in-depth studies of the blood supply to tumors have been conducted using MRI (4-13). Studies using dynamic contrast-enhanced magnetic resonance imaging (DCE-MRI) to investigate breast lesions have been carried out mostly on the qualitative or semi-quantitative analysis level, and those providing a quantitative analysis are relatively few. Quantitative analysis has been performed to evaluate the vascular endothelial permeability and increase in blood flow of tumor tissues for diagnosis, for differential diagnosis and for the evaluation of neoadjuvant chemotherapy for malignant tumors $(14,15)$. This involves analyzing the volume transfer constant (Ktrans), rate constant (Kep), extravascular extracellular volume fraction (Ve), integrated area under the curve (iAUC) and other vascular function parameters, which are of particular interest in the quantitative analysis of DCE-MRI.

In the present study, 3-Tesla (3T) DCE-MRI was used to scan benign and malignant breast lesions, and the differences in vascular function parameters between benign and malignant breast lesions and among various pathological grades of invasive ductal carcinoma quantitatively analyze were quantitatively analyzed.

\section{Materials and methods}

Clinical data. A total of 44 cases admitted to Qianfoshan Hospital of Shandong University between September 2012 and April 2013 were selected. The breast lesions of the cases were diagnosed by physical examination, ultrasound or mammography X-ray detection as well as by breast DCE-MRI examination. All patients were female, aged from 23 to 68 years old with mean age of 41 years. The patients included in the study had no contraindications to MRI, such 
as implanted magnetic devices. Patients who had received any neoadjuvant chemotherapy or who had renal failure were excluded. All patients who underwent MR examination were asked to provide signed informed consent prior to the inspection. Finally, 44 patients (total of 44 lesions) were pathologically confirmed by surgery or biopsy, including 30 cases of malignant tumors $(68.2 \%)$ comprising 26 cases of invasive ductal carcinoma (5 cases of grade I, 14 cases of grade II and 7 cases of grade III), 2 cases of inflammatory breast cancer, 1 case of invasive lipid-rich carcinoma and 1 case of high level ductal carcinoma. The remaining 14 patients had benign tumors (31.8\%), including 5 cases of fibroadenoma, 3 cases of breast hyperplasia with a fibroadenoma formation tendency, 2 cases of breast hyperplasia, 1 case of intraductal papilloma, 1 case of mammary hyperplasia with severe inflammation in certain areas, 1 case of borderline phyllodes tumor and 1 case of lipoma. This study was conducted in accordance with the Declaration of Helsinki. This study was conducted with approval from the Ethics Committee of Shandong University (Jinan, China). Written informed consent was obtained from all participants.

Equipment and parameters. All patients underwent breast MR scanning and T1 dynamic contrast-enhanced MRI. A Siemens Magnetom Skyra 3T MR scanner (Siemens, Erlangen, Germany) and a dedicated eight-channel phased bilateral breast coil were used. The patients were prone, and their bilateral breasts were naturally draped within the coil.

Conventional scanning. Following conventional horizontal, sagittal and coronal positioning and scanning, horizontal position T1 fat-suppression sequences [repetition time (TR)/echo time (TE), $6.0 \mathrm{msec} / 2.62 \mathrm{msec}$; field of view (FOV), $360 \mathrm{~mm}$; matrix, 448x448; slice thickness, $1.2 \mathrm{~mm}$; layer space, $0.24 \mathrm{~mm}$; stimulation number, 1 ; flip angle, $20^{\circ}$ ], horizontal position T2-Dixon sequences (TR/TE 7,210 msec/102 msec; FOV, $340 \mathrm{~mm}$; matrix, 320x320; slice thickness, $4 \mathrm{~mm}$; layer space, $0.8 \mathrm{~mm}$; stimulation number, 1 ; flip angle, $120^{\circ}$ ) and horizontal diffusion bit sequences (TR/TE, 4,300 msec/63 msec; FOV, $340 \mathrm{~mm}$; matrix, 220x220; slice thickness, $5 \mathrm{~mm}$; layer space, $1 \mathrm{~mm}$; stimulation number, 4 times) were performed.

T1 dynamic contrast-enhanced scanning. Original T1 scanning (T1 mapping) was initially performed, followed by an enhanced T1 line sequential scan. T1 map scanning was performed by multi-flip angle technology with the following parameters: TR/TE, $7.84 \mathrm{msec} / 3.37 \mathrm{msec}$; FOV, $340 \mathrm{~mm}$; matrix, 224x224; slice thickness, $1.5 \mathrm{~mm}$; layer space, $0.3 \mathrm{~mm}$; stimulation number, 1 ; flip angle, $3 \% 16^{\circ}$. The continuous T1-enhanced sequence scanning parameters were as follows: TR/TE, $5.61 \mathrm{msec} / 1.74$ msec; FOV, 340 mm; matrix, 224x224; slice thickness, $1.5 \mathrm{~mm}$; layer space, $0.3 \mathrm{~mm}$; stimulation number, 1; flip angle, $10^{\circ}$; total scan time, 22 times; single phase scan time, $30.1 \mathrm{sec}$ with total time of $11.15 \mathrm{~min}$. Following the second phase data collection, $20 \mathrm{ml}$ gadolinium diethylenetriaminepentacetate (DTPA; Haibo Lecco Xinyi Pharmaceutical Co., Ltd., Shanghai, China) contrast agent was intravenously injected at high pressure, followed by a 20-ml injection of saline at a flow rate of $5 \mathrm{ml} / \mathrm{sec}$. Then, 20 phases were continuously collected.
Data processing and analysis. The scanned images were transmitted to Siemens workstation SYGNO VE40A; post-processing work was performed using TISSUE 4D software. The most evident breast artery or internal thoracic artery was selected to obtain the arterial input function (AIF). The vascular function parameters of the lesions were measured by selecting a region of interest (ROI), as shown on the pseudo-color images.

ROI selection. The substantive component of a mass was selected as the region of interest, and three levels were selected for each lesion (the level of the maximum cross-sectional area, and one at each of the upper and lower levels), avoiding necrotic tissue, voids, vascular calcification and other features. For each vascular permeability parameter, the average of the parameter at the three levels was taken as the vascular permeability parameter of the lesion.

Statistical analysis. SPSS software version 7.0 (SPSS, Inc., Chicago, IL, USA) was used to conduct the statistical analysis. Data are expressed as mean \pm standard deviation. The vascular function parameters between benign and malignant tumors were compared using Student's t-test or adjusted t-test. The vascular function parameters among different pathological grades of invasive ductal breast cancers were compared using repeated measures analysis of variance. $\mathrm{P}<0.05$ was considered to indicate a statistically significant difference.

\section{Results}

Differences in Ktrans, Kep and iAUC between benign and malignant breast lesions. The Ktrans, Kep and iAUC values of the malignant breast lesions were significantly higher than those of the benign lesions, and had slight differences between the different levels of invasive ductal carcinoma. Malignant breast lesions generally were significantly enhanced in the early enhancement stage, and red (representing higher perfusion) was observed in lesion regions of the dynamic contrast-enhanced pseudo-color maps; the higher the value of perfusion parameters, the larger the relative scope of the red zone and the deeper the color. In addition, the higher the relative value of the high perfusion parameters, the deeper the invasive ductal carcinoma malignancy degree (represented in black and white in Figs. 1-3). The benign breast lesions generally exhibited delayed enhancement, and yellow (representing lower perfusion) was observed in lesion regions of the dynamic contrast-enhanced pseudo-color maps (represented in black and white in Fig. 4).

Significance of vascular function parameters in the differential diagnosis of benign and malignant breast lesions. The differences in Ktrans, Kep and iAUC between benign and malignant tumors were statistically significant $(\mathrm{P}<0.05)$. The difference in Ve between benign and malignant tumors was not statistically significant $(\mathrm{P}>0.05$, Table I).

Significance of vascular function parameters in the grading of breast invasive ductal carcinoma. The differences in Ktrans, Kep and iAUC between grade I and grade II, and between grade I and grade III of ductal carcinoma were 


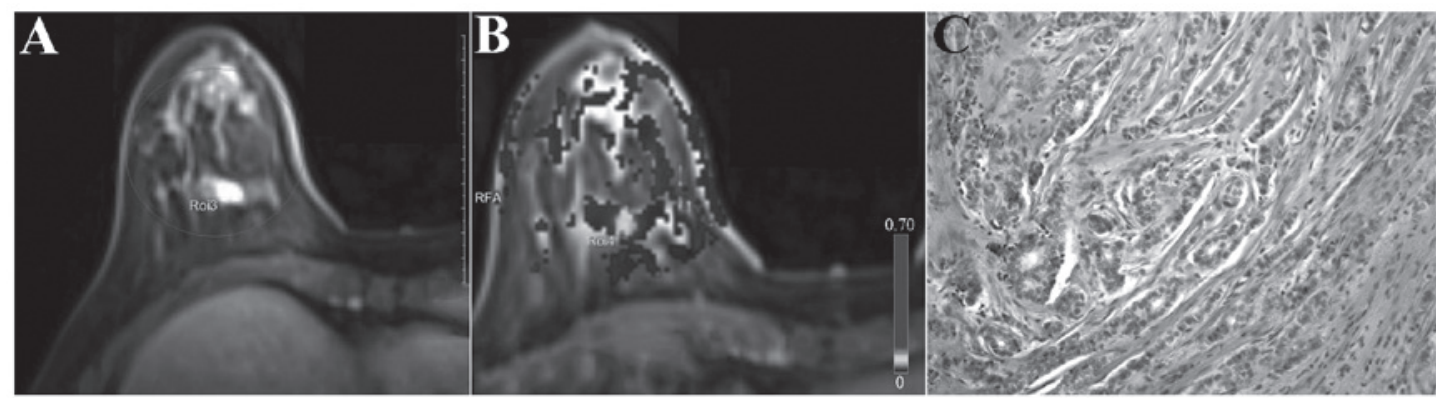

Figure 1. Invasive ductal carcinoma of grade I in the right breast. (A) Early dynamic contrast-enhanced image, (B) image of vascular function parameters and (C) pathological image (hematoxylin and eosin stained; magnification, x200).

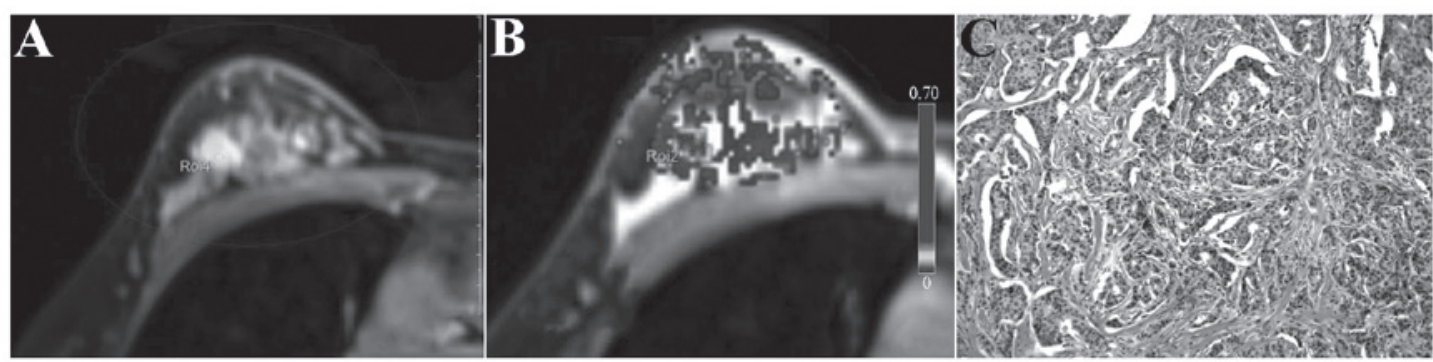

Figure 2. Invasive ductal carcinoma of grade II in the right breast. (A) Early dynamic contrast-enhanced image, (B) image of vascular function parameters and (C) pathological image (hematoxylin and eosin stained; magnification, x200).
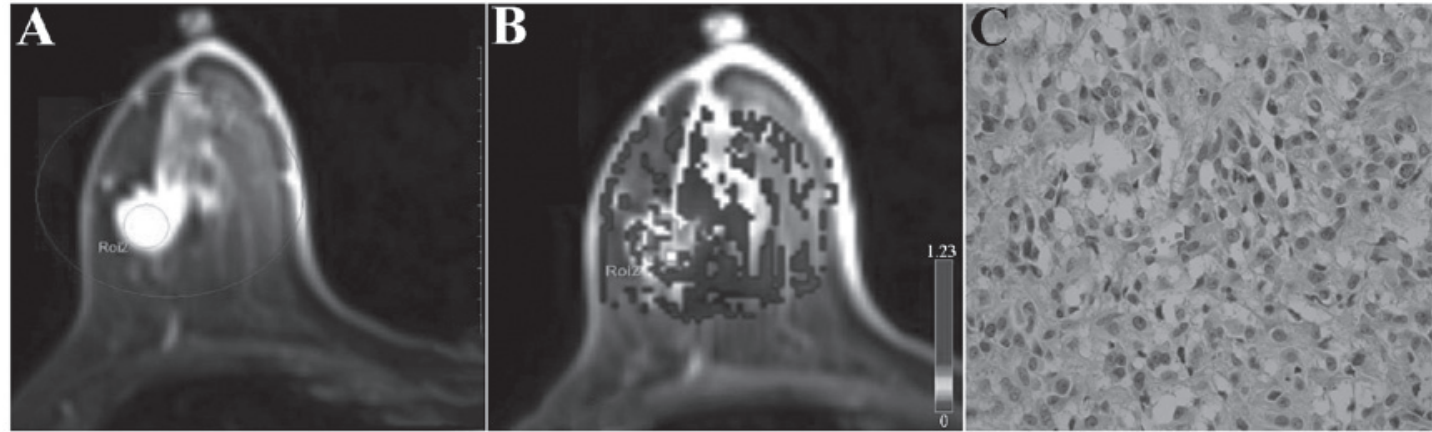

Figure 3. Invasive ductal carcinoma of grade III in the right breast. (A) Early dynamic contrast-enhanced image, (B) image of vascular function parameters and (C) pathological image (hematoxylin and eosin stained; magnification, x200).
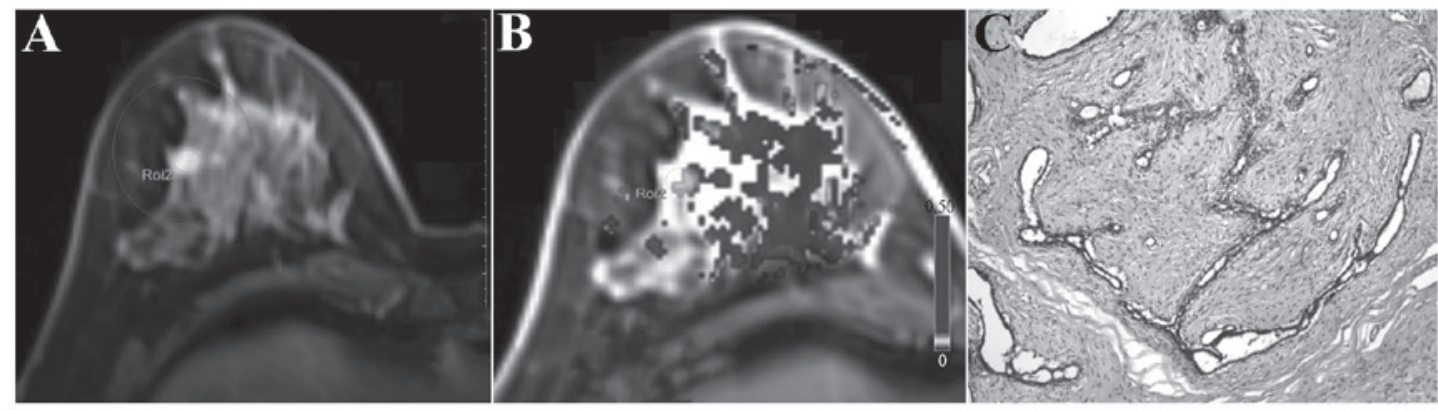

Figure 4. Fibroadenoma in the right breast. (A) Late dynamic contrast-enhanced image, (B) image of vascular function parameters and (C) pathological image (hematoxylin and eosin stained; magnification, x200).

statistically significant $(\mathrm{P}<0.05)$. The differences in Ktrans and Kep between grade II and grade III of invasive ductal carcinoma were not statistically significant $(\mathrm{P}>0.05)$. No statistically significant differences in Ve were observed among grades I, II and III of invasive ductal carcinoma $(\mathrm{P}>0.05$, Table II $)$. 
Table I. Comparison of vascular function parameters between benign and malignant groups.

\begin{tabular}{lccccc}
\hline Group & $\mathrm{n}$ & Ktrans $\left(\mathrm{min}^{-1}\right)$ & Kep $\left(\mathrm{min}^{-1}\right)$ & Ve & iAUC \\
\hline Benign & 14 & $0.136 \pm 0.088$ & $0.291 \pm 0.185$ & $0.565 \pm 0.196$ & $4.633 \pm 3.687$ \\
Malignant & 30 & $0.967 \pm 0.361^{\mathrm{a}}$ & $1.742 \pm 0.552^{\mathrm{a}}$ & $0.581 \pm 0.137$ & $14.614 \pm 5.692^{\mathrm{a}}$ \\
\hline
\end{tabular}

Values shown are mean standard \pm deviation. ${ }^{\mathrm{a}} \mathrm{P}<0.05$ vs. the benign group. Ktrans, volume transfer constant; Kep, rate constant; Ve, extravascular extracellular volume fraction; iAUC, integrated area under the curve.

Table II. Comparison of vascular function parameters among different histological grades of invasive ductal carcinoma.

\begin{tabular}{lrcccc}
\hline Grading & $\mathrm{n}$ & Ktrans $\left(\mathrm{min}^{-1}\right)$ & Kep $\left(\mathrm{min}^{-1}\right)$ & Ve & iAUC \\
\hline Grade I & 5 & $0.579 \pm 0.030$ & $1.199 \pm 0.050$ & $0.480 \pm 0.016$ & $7.737 \pm 0.170$ \\
Grade II & 14 & $1.196 \pm 0.268^{\mathrm{a}}$ & $2.000 \pm 0.503^{\mathrm{a}}$ & $0.628 \pm 0.118$ & $14.558 \pm 4.054^{\mathrm{a}}$ \\
Grade III & 7 & $1.011 \pm 0.242^{\mathrm{a}}$ & $2.001 \pm 0.312^{\mathrm{a}}$ & $0.556 \pm 0.199$ & $20.465 \pm 1.936^{\mathrm{a}}$
\end{tabular}

Values shown are mean standard \pm deviation. ${ }^{\mathrm{a}} \mathrm{P}<0.05$ vs. grade I. Ktrans, volume transfer constant; Kep, rate constant; Ve, extravascular extracellular volume fraction; iAUC, integrated area under the curve.

Table III. Efficacy of Ktrans, Kep and iAUC in the diagnosis of benign and malignant lesions.

\begin{tabular}{lccc}
\hline Parameters $(\%)$ & Boundary value & Sensitivity (\%) & Specificity $(\%)$ \\
\hline Ktrans $\left(\mathrm{min}^{-1}\right)$ & 0.832 & 80.0 & 92.9 \\
Kep $\left(\mathrm{min}^{-1}\right)$ & 1.536 & 66.7 & 86.7 \\
iAUC & 12.488 & 73.3 & 85.7
\end{tabular}

Ktrans, volume transfer constant; Kep, rate constant; iAUC, integrated area under the curve.

Diagnostic value of vascular function parameters at $95 \%$ confidence. The lower bounds at $95 \%$ confidence of the Ktrans, Kep and iAUC values in malignant cancers were defined as the boundary values for the respective differential diagnosis of benign and malignant lesions. Ktrans, Kep and iAUC had high sensitivity and specificity in the differential diagnosis of benign and malignant lesions, of which Ktrans had the superior diagnostic performance with the highest sensitivity and specificity (Table III)

\section{Discussion}

Perfusion-weighted imaging (PWI) is an imaging technique that reflects tissue or microvascular lesion distribution and blood flow perfusion, which is able to evaluate angiogenesis in tumor tissues in vivo in a non-destructive manner and is extremely valuable for studying tumor blood supply (16). DCE-MRI is a PWI technology that can indirectly reflect the functional status of tumor blood vessels, thereby providing a reliable basis for further diagnosis and treatment by revealing the microvascular perfusion status and the degree of tissue vascularization. The pathological basis of the perfusion effect includes changes in the number of tumor vascular vessels, and changes in blood vessel function. Malignant tumors can release vascular endothelial cell growth factor to induce capillary growth. For such tumors, it has been observed that neovessel density is high, an early tumor perfusion effect is evident, neovascular walls are incomplete, and contrast agent rapidly bleeds into the extracellular space to result in rapid filling of the tumor with contrast agent and a fast outflow rate (17). However, for benign tumors, vascular provision is relatively less, and the contrast agent infusion effect is not evident (18).

DCE-MRI can dynamically display the pharmacokinetic changes of contrast agent in the vessels as a continuously obtained series of images. Currently, the blood dual-chamber kinetic model (dual-chamber representation of the microvascular and extravascular interstitium) proposed by Tofts et al (19) is widely used to perform DCE-MRI. The results are analyzed to obtain the following vascular function parameters: Ktrans, Kep, Ve and iAUC. iAUC is actually a semi-quantitative parameter that is associated with blood flow into the tumor, tumor perfusion and tumor tissue spaces $(20,21)$, and can effectively reflect changes in Ktrans, Kep and Ve.

Ocak et al (22) demonstrated that the Ktrans and Kep values of prostate malignancy were significantly higher than those of benign lesions. Yao et al (23) identified that Ktrans and Kep differed significantly between normal bowel walls and rectal cancer. In addition, the authors observed statistically significant differences in Ktrans between colorectal cancer with lymph node metastasis and that 
without lymph node metastasis, and among different Dukes stage of colorectal cancer, while Kep and Dukes' stage had a moderate correlation. Li et al (24) found that Kep was able to predict the efficacy of the first cycle of neoadjuvant chemotherapy in breast cancer patients. Nilsen et al (25) observed that Ktrans and Kep had a statistically significant difference between breast cancer lesions associated with bone metastasis and that without bone metastases; the perfusion and vascular permeability of breast cancer lesions with metastasis were significantly lower than those of non-metastatic breast cancer lesions. The present study demonstrated that the Ktrans, Kep and iAUC values of malignant breast lesions were significantly higher than those of benign lesions; this may be associated with the biological characteristics of the lesions. The malignant lesions were vigorously growing with increased tumor angiogenesis, increased microvessel density and structural disorder, partially incomplete neovascular endothelium and abnormal vascular endothelial cell morphology. They also exhibited enlarged spaces between the endothelium and basement membrane, basement membrane and vascular pericytes. Thus, the angiogenesis of malignant lesions was associated with a high permeability (26). For benign lesions, the neovascularization was less than that of malignant lesions, and vascular endothelium growth was relatively complete. Therefore, for malignant lesions, the diffusion rate of contrast agent from intravascular to extravascular tissues and the diffusion rate of contrast agent from extravascular tissues back to vessels were significantly greater than those of benign lesions, which was reflected as higher Ktrans and Kep values for malignant lesions than for benign lesions.

Tofts (27) found that the Ve value was less consistent than the other vascular function parameters; this was considered to be due with the fact that Ve is often affected by edema around the lesion. Certain scholars also consider that this may be associated with the slow rate of change in the relative proportions of extravascular extracellular volume within the organization in the process of disease development, resulting in the Ve value range between benign and malignant lesions having a degree of overlap $(28,29)$. The present study demonstrated that $\mathrm{Ve}$ values did not significantly differ between benign and malignant lesions, which is associated the characteristics of Ve, as it represents the percentage of contrast agent retained in tissue spaces. Ktrans, Kep and Ve satisfy the following relationship: $\mathrm{Ve}=\mathrm{Ktrans} / \mathrm{Kep}$. The neovascular permeability of malignant lesions is high, resulting in increased Ktrans and Kep values; however, the increase in the proportions of the two values is uncertain. Therefore, the Ve values of benign and malignant lesions did not exhibit a significant difference.

The study further analyzed the vascular function parameters among different pathological grades in invasive ductal carcinoma. It was found that the differences in Ktrans and Kep among grades I, II and III of invasive ductal carcinoma were statistically significant, while no statistically significant differences of Ktrans and Kep were observed between grades II and III of invasive ductal carcinoma. The Ve values demonstrated no statistically significant differences among grades I, II and III of invasive ductal carcinoma. Tumor formation and development can be divided into two stages, namely the clonal proliferation stage of tumor cells and the tumor angiogenesis-promoting sustainable growth phase (30). Breast cancer is pathologically graded through duct/gland formation, nuclear pleomorphism and mitotic count. Grade I invasive ductal carcinoma comprises mostly duct/gland formation and early mitotic stage. At this grade, the endothelial integrity is relatively good. The vascular endothelial differentiation of grade II and III invasive ductal carcinoma is poor with high permeability and increased perfusion resistance; however, as grades II and III are characterized by high permeability, there may be some overlap in the monitoring parameters of tumor blood vessel growth of the two grades. Furthermore, the number of cases was few, which may lead to the generation of errors.

Baek et al (31) found that Ktrans had relatively greater significance among the vascular function parameters for the differential diagnosis of benign and malignant lesions. The present study found that the diagnostic sensitivity was $80.0 \%$ when Ktrans was $0.832 \mathrm{~min}^{-1}$, and the specificity was $92.9 \%$, both of which were higher than the values for Kep and iAUC. Therefore, Ktrans was more meaningful in differentiating benign and malignant lesions, and can provide a quantitative indicator for clinical diagnosis.

In conclusion, this study compared vascular function parameters between benign and malignant breast lesions and among different levels of invasive ductal carcinoma and found that vascular function parameters (Ktrans, Kep and iAUC) were meaningful in the differential diagnosis of benign and malignant breast lesions. The sensitivity and specificity of Ktrans were the highest, and had a certain degree of significance in the grading of invasive ductal carcinoma. However, this study included a relatively small number of patients; a larger sample should be investigated in future studies. The optimal imaging parameters of dynamic contrast-enhanced MR imaging of the breast were collected and analyzed to obtain optimal vascular function parameters. Thus, the diagnosis and differential diagnosis of benign and malignant breast lesions was investigated.

\section{References}

1. Berg WA, Gutierrez L, NessAiver MS, Carter WB, Bhargavan M, Lewis RS and Ioffe OB: Diagnostic accuracy of mammography, clinical examination, US, and MR imaging in preoperative assessment of breast cancer. Radiology 233: 830-849, 2004.

2. Liberman L: Breast cancer screening with MRI - what are the data for patients at high risk? N Engl J Med 351: 497-500, 2004.

3. Folkman J: Tumor angiogenesis: therapeutic implications. N Engl J Med 285: 1182-1186, 1971.

4. Weidner N, Folkman J, Pozza F, et al: Tumor angiogenesis: a new significant and independent prognostic indicator in early-stage breast carcinoma. J Natl Cancer Inst 84: 1875-1887, 1992.

5. DeVries A, Griebel J, et al: Perfusion-index values evaluated by dynamic magnetic resonance imaging in advanced rectal carcinoma. A new predictor of response to preoperative radiochemotherapy? Strahlenther Onkol 176: 567-572, 2000 (In German).

6. Kremer S, Grand S, Rémy C, et al: Contribution of dynamic contrast MR imaging to the differentiation between dural metastasis and meningioma. Neuroradiology 46: 642-648, 2004.

7. Haris M, Gupta RK, Singh A, et al: Differentiation of infective from neoplastic brain lesions by dynamic contrast-enhanced MRI. Neuroradiology 50: 531-540, 2008.

8. Zhang H, Rödiger LA, et al: Perfusion MR imaging for differentiation of benign and malignant meningiomas. Neuroradiology 50: 525-530, 2008.

9. Pickles MD, Manton DJ, Lowry M and Turnbull LW: Prognostic value of pre-treatment DCE-MRI parameters in predicting disease free and overall survival for breast cancer patients undergoing neoadjuvant chemotherapy. Eur J Radiol 71: 498-505, 2009. 
10. Kang TW, Kim ST, Byun HS, et al: Morphological and functional MRI, MRS, perfusion and diffusion changes after radiosurgery of brain metastasis. Eur J Radiol 72: 370-380, 2009.

11. Hauser T, Essig M, Jensen A, et al: Characterization and therapy monitoring of head and neck carcinomas using diffusion-imagingbased intravoxel incoherent motion parameters-preliminary results. Neuroradiology 55: 527-536, 2013.

12. Larsen VA, Simonsen HJ, Law I, Larsson HB and Hansen AE: Evaluation of dynamic contrast-enhanced T1-weighted perfusion MRI in the differentiation of tumor recurrence from radiation necrosis. Neuroradiology 55: 361-369, 2013.

13. Bäuerle T, Seyler L, Münter M, et al: Diffusion-weighted imaging in rectal carcinoma patients without and after chemoradiotherapy: a comparative study with histology. Eur J Radiol 82: 444-452, 2013

14. Jones EF, Sinha SP, Newitt DC, Klifa C, Kornak J, Park CC and Hylton NM: Mri enhancement in stromal tissue surrounding breast tumors: association with recurrence free survival following neoadjuvant chemotherapy. PLoS One 8: e61969, 2013.

15. Li X, Arlinghaus LR, Ayers GD, et al: Dce-mri analysis methods for predicting the response of breast cancer to neoadjuvant chemotherapy: pilot study findings. Magn Reson Med 71: 1592-1602, 2014.

16. Schnall MD, Blume J, Bluemke DA, DeAngelis GA, et al: Diagnostic architectural and dynamic features at breast MR imaging: multicenter study. Radiology 238: 42-53, 2006.

17. Rykala J, Przybylowska K, Majsterek I, et al: Angiogenesis markers quantification in breast cancer and their correlation with clinicopathological prognostic variables. Pathol Oncol Res 17: 809-817, 2011.

18. Medeiros LR, Duarte CS, Rosa DD, Edelweiss MI, et al: Accuracy of magnetic resonance in suspicious breast lesions: a systematic quantitative review and meta-analysis. Breast Cancer Res Treat 126: 273-285, 2011.

19. Tofts PS, Brix G, Buckley DL, et al: Estimating kinetic parameters from dynamic contrast-enhanced t1-weighted mri of a diffusable tracer: standardized quantities and symbols. J Magn Reson Imaging 10: 223-232, 1999.

20. Padhani AR and Husband JE: Dynamic contrast-enhanced mri studies in oncology with an emphasis on quantification, validation and human studies. Clin Radiol 56: 607-620, 2001.
21. Preda A, van Vliet M, Krestin GP, Brasch RC and van Dijke CF: Magnetic resonance macromolecular agents for monitoring tumor microvessels and angiogenesis inhibition. Invest Radiol 41: 325-331, 2006

22. Ocak I, Bernardo M, Metzger G, Barrett T, Pinto P, Albert PS and Choyke PL: Dynamic contrast-enhanced mri of prostate cancer at 3 t: a study of pharmacokinetic parameters. AJR Am J Roentgenol 189: 849-858, 2007.

23. Yao WW, Zhang H, Ding B, et al: Rectal cancer: 3d dynamic contrast-enhanced mri; correlation with microvascular density and clinicopathological features. Radiol med 116: 366-374, 2011.

24. Li X, Arlinghaus LR, Ayers GD, et al: dce-mri analysis methods for predicting the response of breast cancer to neoadjuvant chemotherapy: pilot study findings. Magn Reson Med 71: 1592-1602, 2014.

25. Nilsen LB, Fangberget A, Geier OM, Engebraaten O, Borgen E, Olsen DR and Seierstad T: Associations between tumor vascularization assessed by in vivo dce-mri and the presence of disseminated tumor cells in bone marrow in breast cancer patients at the time of diagnosis. J Magn Reson Imaging Jan 27, 2014 (Epub ahead of print).

26. Jain RK: Normalization of tumor vasculature: an emerging concept in antiangiogenic therapy. Science 307: 58-62, 2005.

27. Tofts PS: Modeling tracer kinetics in dynamic gd-dtpa mr imaging. J Magn Reson Imaging 7: 91-101, 1997.

28. Wang Y1, Huang W, Panicek DM, et al: Feasibility of using limited-population-based arterial input function for pharmacokinetic modeling of osteosarcoma dynamic contrast-enhanced MRI data. Magn Reson Med 59: 1183-1189, 2008.

29. Koo HR, Cho N, Song IC, et al: Correlation of perfusion parameters on dynamic contrast-enhanced MRI with prognostic factors and subtypes of breast cancers. J Magn Reson Imaging 36: $145-151,2012$.

30. Siegmann KC, Müller-Schimpfle M, Schick F, et al: Mr imaging-detected breast lesions: histopathologic correlation of lesion characteristics and signal intensity data. Am J Roentgenol 178: 1403-1409, 2002.

31. Baek HM, Chen JH, Nie K, et al: Predicting pathologic response to neoadjuvant chemotherapy in breast cancer by using $\mathrm{mr}$ imaging and quantitative $1 \mathrm{~h} \mathrm{mr}$ spectroscopy. Radiology 251 : 653-662, 2009. 